Tropical Journal of Pharmaceutical Research June 2017; 16 (6): 1437-1442

ISSN: $1596-5996$ (print); 1596-9827 (electronic)

(C) Pharmacotherapy Group, Faculty of Pharmacy, University of Benin, Benin City, 300001 Nigeria.

All rights reserved.

Available online at http://www.tjpr.org

Original Research Article

http://dx.doi.org/10.4314/tjpr.v16i6.31

\title{
Comparison of the therapeutic effects of continuous hyperthermic peritoneal perfusion with systemic intravenous chemotherapy
}

\author{
Chao Li ${ }^{1}$, Chun-Hua Dong ${ }^{2}$, Hong-Na Lv ${ }^{1}$ and Xiao-Dan Liu ${ }^{3 \star}$ \\ ${ }^{1}$ Department of Gastroenterology, ${ }^{2}$ Department of Gynecology, Binzhou People's Hospital, Binzhou, 256610, ${ }^{3}$ Department of \\ Hematology, Binzhou Medical University Hospital, Binzhou, 256603, PR China
}

*For correspondence: Email: liuxdtx@163.com

Sent for review: 9 January 2017

Revised accepted: 13 May 2017

\begin{abstract}
Purpose: To compare the effectiveness and safety of intravenous cisplatin (DDP) and 5-fluorouracil (5FU) alone or in combination with continuous hyperthermic peritoneal perfusion (CHPP) for the treatment of malignant ascites.

Methods: In the study, 124 patients with tumour-induced malignant ascites were assign to test and control groups according to the sequence of their hospital visits. Patients in the test group were treated with intravenous DDP and 5-FU combined with CHPP, whereas patients in the control group were only treated with DDP and 5-FU. The treatments in both groups lasted for 4 weeks. Thereafter, treatment efficacy, remission of abdominal distension, ascites, Karnofsky Performance Scale (KPS) score, and incidence of adverse reactions were compared between the two groups.

Results: The overall response rates of the test and control groups were 85.50 (53/62) and $35.50 \%$ (22/62), respectively, and the difference was significant $(p<0.05)$. Patients in the test group showed significant reduction in abdominal distension and markedly reduced ascites compared to the controls. The improvement in KPS score was superior in the test group $(p<0.05)$. No treatment-associated complications, such as intestinal adhesions or obstruction and grade III or IV toxic and side reactions, were found in either group. The incidence of adverse reactions was lower in the test group than in the controls.

Conclusion: Chemotherapy in combination with CHPP is safe and effective for patients with advanced malignant tumours and ascites should be promoted clinically.
\end{abstract}

Keywords: Malignant ascites, Continuous hyperthermic peritoneal perfusion, Systemic intravenous chemotherapy

Tropical Journal of Pharmaceutical Research is indexed by Science Citation Index (SciSearch), Scopus, International Pharmaceutical Abstract, Chemical Abstracts, Embase, Index Copernicus, EBSCO, African Index Medicus, JournalSeek, Journal Citation Reports/Science Edition, Directory of Open Access Journals (DOAJ), African Journal Online, Bioline International, Open-J-Gate and Pharmacy Abstracts

\section{INTRODUCTION}

Malignant ascites refers to abnormally increased liquid in the abdomen induced by diffuse lesions caused by systemic or abdominal malignancies $[1,2]$. When there is little ascites, subjective symptoms are not obvious, whereas more ascites can induce symptoms such as abdominal distension, stomach ache, shortness of breath, gastrointestinal dysfunction, and even intestinal obstruction, which can severely affect the patient's quality of life $[3,4]$. Although the survival of patients with malignant tumours is low, successful treatment can improve the prognosis of patients with malignant ascites.

There are many treatments for malignant ascites; however, each has limitations. Surgery and 
radiotherapy cannot effectively eradicate large lesions, and intravenous chemotherapy is ineffective when using drugs that have low concentrations in the abdominal cavity $[5,6]$. Some researchers have developed a new treatment, namely continuous hyperthermic peritoneal perfusion (CHPP), which is based on the different tolerance of tumour and normal cells to temperature and the synergistic effects of thermo-chemotherapy [7]. CHPP can prevent tumour recurrence by killing free tumour cells in the abdominal cavity and small metastases. To investigate the safety and effectiveness of CHPP, this retrospective study analysed 124 cases of malignant ascites seen at Binzhou People's Hospital, Shandong, China, and compared the safety and effectiveness of CHPP compared to systemic intravenous chemotherapy.

\section{METHODS}

\section{Subjects}

The study enrolled 124 patients who had malignant tumour-induced malignant ascites and were admitted to Binzhou People's Hospital from December 2013 to December 2015. They were randomly divided into test and control groups according to the admission time. The test group comprised 30 males and 32 females, with a median age of 60 (range 44 - 76) years; there were 25 cases of ovarian cancer, 16 cases of gastric carcinoma, 12 cases of liver cancer, 6 cases of oesophageal carcinoma, and 3 cases of intestinal cancer. The control group comprised 27 males and 35 females, , with a median age of 58 (range 42 - 71) years; there were 22 cases of gastric carcinoma, 20 cases of ovarian cancer, 7 cases of intestinal cancer, and 6 cases of oesophageal carcinoma. The differences in sex, age, and disease between the two groups were not significant $(p>0.05)$. The study was approved by the Medical Ethics Committee of Binzhou People's Hospital (approval no. BZPH201602018LC), and the experiment followed the guidelines of the Declaration of Helsinki [8].

\section{Inclusive and exclusive criteria}

\section{Inclusive criteria}

The study included patients who were at least 18 years old; had pathologically or cytologically confirmed malignant tumours and malignant ascites; had indications for systemic chemotherapy; accepted the first-line chemotherapy regimen; were diagnosed with uncoated ascites $(\geq 5 \mathrm{~cm}$ ) by $B$ ultrasound or computed tomography; had a Karnofsky performance scale (KPS) score of at least 60; had predicted survival exceeding 4 weeks; had no severe cardiac, hepatic, or pulmonary dysfunction; had no contraindications to chemotherapy; agreed to the release of relevant treatment data; and had been informed of the risks of the study and provided informed consent.

\section{Exclusion criteria}

Patients were excluded if they had a KPS score less than 60 points; could not tolerate chemotherapy; had undergone chemotherapy or specific treatment in the 4 weeks before this study; had poor bone marrow function or severe anaemia; had white blood cell and platelet counts below the normal levels; were suffering from intestinal obstruction, peritoneal adhesions, or intra-abdominal infection; had ascites induced by liver cirrhosis or other diseases; failed to follow the treatment plans formulated by doctors; or could not control their own behaviours and language because of diseases such as mental disturbance and dysgnosia.

\section{Treatment methods}

\section{Intravenous chemotherapy}

Patients in the control group were treated with systemic intravenous chemotherapy comprising cisplatin (CDDP; Jinzhou Jiutai Pharmaceutical Co., Ltd., China; Batch no. 21020212) at a dose of $100 \mathrm{mg} / \mathrm{m}^{2}$, once every 3 weeks and 5fluorouracil (5-FU; Shanghai Xudong Pharmaceutical Co. Ltd, China, Batch no. H31020593) 500 mg each time, once a week, with a 2 - week interval after every 4 - week cycle. In addition, potassium chloride, mannitol, and furosemide were used for hydration and dieresis; the urine volume was controlled at 2000 $\mathrm{mL} / \mathrm{d}$. Abdominal puncture and tapping were performed under ultrasound guidance once every 2 days. The tapping rate was kept between 1500 and $2000 \mathrm{~mL}$. This treatment plan was used three or four times as tolerated by the patients.

\section{CHPP}

Patients in the test group were treated with CHPP. With the patient in a supine position supported by a pillow, the left and right upper and lower abdomen were examined by $B$ ultrasound. Sites with sufficient ascites and no adhesions between the abdominal wall and organs and tissues in the abdominal cavity were selected for puncture. The punctures were kept away from any original abdominal wounds. The puncture sites were disinfected. After local 
anaesthesia using $0.5 \%$ lidocaine, a $1 \mathrm{~cm}$ long incision was made at each puncture site and a 1 $\mathrm{cm}$ diameter trocar was inserted into the abdominal cavity. Then perfusion catheters were inserted into the left and right upper abdomen, and outflow catheters were inserted into the left and right lower abdomen; the insertion lengths were about $80 \mathrm{~cm}$. Next, $1500 \mathrm{mg} \mathrm{5-FU} \mathrm{and} 100$ mg DDP were added to 2500 - $4500 \mathrm{~mL}$ normal saline. The amount of normal saline was adjusted to the volume of ascites such that the perfusion liquid could be pumped through the closed pipeline system. The perfusion was controlled at a speed of $500 \mathrm{~mL} / \mathrm{min}$ at $41.5^{\circ} \mathrm{C} \pm$ $0.2^{\circ} \mathrm{C}$, for $60-90 \mathrm{~min}$. To relieve abdominal pain and increase the sensitivity to thermotherapy, $100 \mathrm{mg}$ of $2 \%$ lidocaine was added. To prevent gastrointestinal side effects, an anti-emetic, dexamethasone, and Vitamin $\mathrm{B}_{6}$ were administered $30 \mathrm{~min}$ before perfusion. To relieve abdominal distension, some of the perfusion liquid was removed after each perfusion. The patients were given diuretics, intravenous infusions, and supportive treatment such as supplemental albumin and plasma. This treatment was performed once a week, for 4 weeks.

\section{Treatment indices}

\section{Cure}

According to the World Health Organization Response Evaluation Criteria in Solid Tumours [9], complete disappearance of ascites for more than 4 weeks is considered complete remission (CR), a one-half or greater reduction in the ascites for more than 4 weeks is considered partial remission (PR), a reduction in the ascites of less than one-half or no obvious changes is considered no remission (NR), and increased ascites or aggravated symptoms are deemed progressive disease (PD). The overall response rate was calculated as:

$R=(a+b) / N * 100 \%$

where $R$ is the overall response rate, $a$ is cases of $C R, b$ is cases of PR, and $N$ is the total number of cases. The abdominal distention was scored before treatment and 1 week after treatment as follows: severe abdominal distension that affected breathing, 3 points; severe abdominal distension that had no influence on breathing, 2 points; abdominal distension that had no influence on life, 1 point; and no abdominal distension, 0 points. The depth of fluid sonolucent areas was examined by abdominal B ultrasound before and 1 week after treatment and compared.

\section{KPS score}

The KPS scores before treatment and 4 weeks after treatment were compared between the two groups. An increase in 10 or more points compared to the score before treatment indicated improved quality of life; an increase or decrease in no more than 10 points was deemed stable quality of life; and a decrease in more than 10 points was deemed a reduced quality of life.

\section{Adverse reactions}

The National Cancer Institute Common Toxicity Criteria [10] were used to evaluate the state of different systems after treatment, and to determine the toxic and side effects on different systems. Signs of intestinal obstruction, intestinal adhesions, gastrointestinal symptoms (nausea, emesis, abdominal distension, and stomach ache), fever, lack of strength, diaphoresis, intestinal paralysis, white blood cell reduction, myelosuppression were evaluated.

\section{Statistical analysis}

The data were analysed statistically using SPSS ver. 20.0. Enumeration data are expressed as the rate. The results are reported as mean \pm standard deviation (SD). Groups were compared to the chi-square test or $t$-test as appropriate. Differences were considered statistically significant if $p<0.05$.

\section{RESULTS}

\section{Curative effects seen in both groups}

The overall response rate of the test group was $85.50 \%$, including 37 cases of CR, 16 cases of $\mathrm{PR}, 6$ cases of NR, and 3 cases of PD. The overall response rate of the control group was $35.50 \%$, including 13 cases of CR, 9 cases of PR, 26 cases of NR, and 14 cases of PD. The overall response rate of the test group was higher than that of the control group $(p<0.05)$.

\section{Improvement in abdominal distension and ascites changes in both groups}

Before treatment, the difference in the abdominal distension scores of the two groups was not significant. After treatment, the abdominal distension scores improved in both groups, but the improvement in the test group was superior to that in the control group ( $p<0.05$; Table 1$)$. Before treatment, the depth of ascites was similar in both groups; after treatment, the depth of ascites decreased in both groups, and the 
Table 1: Comparison of the abdominal distension scores and ascites in the two groups before and after treatment (mean \pm SD)

\begin{tabular}{lccc}
\hline Variable & & Test group & Control group \\
\hline $\begin{array}{l}\text { Abdominal distention score } \\
\text { (points) }\end{array}$ & Before & $2.42 \pm 0.71$ & $2.53 \pm 0.79$ \\
& After & $0.87 \pm 0.53^{* \#}$ & $1.89 \pm 0.64^{\#}$ \\
Ascites $(\mathrm{cm})$ & Before & $7.29 \pm 0.51$ & $2.38 \pm 0.62$ \\
& After & $1.87 \pm 2.49^{\#}$ & $4.91 \pm 2.57^{\#}$ \\
\hline Notes: ${ }^{*} P<0.05$ compared to the control group: ${ }^{\#} p<0.05$ compared to before treatment
\end{tabular}

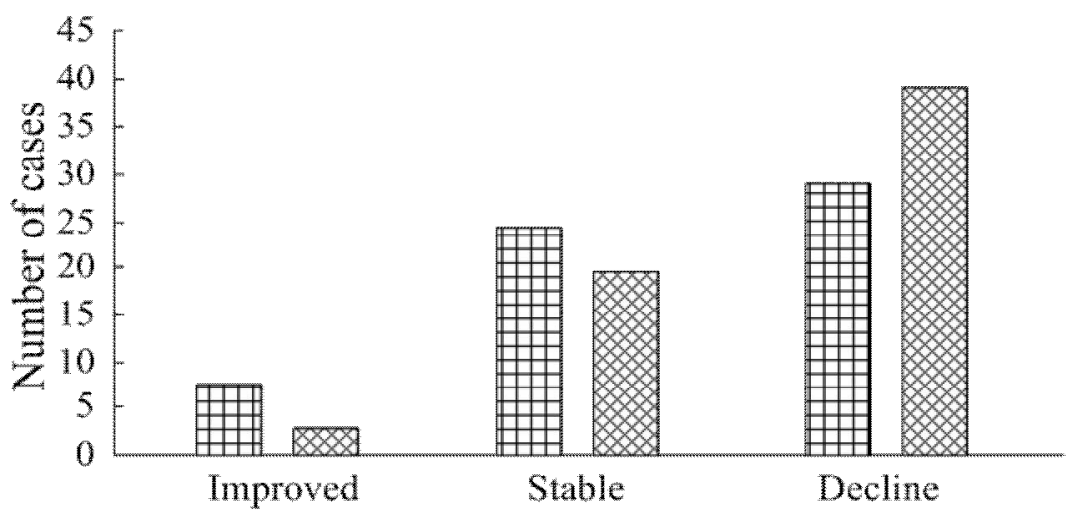

田Test group $\otimes$ Control group

Figure 1: KPS scores

Table 2: Incidence of adverse reactions $\{N(\%)\}$

\begin{tabular}{lcc}
\hline Variable & Test group & Control group \\
\hline Nausea and vomiting & $52(83.9)$ & $41(66.1)$ \\
Fever, lack of strength and diaphoresis & $30(48.4)$ & $22(35.5)$ \\
Abdominal distension and pain & $9(14.5)$ & $5(8.1)$ \\
Intestinal paralysis and obstruction & $0(0.0)$ & $0(0.0)$ \\
Decreased white blood cells & $43(69.4)^{*}$ & $62(100.0)^{*}$ \\
Myelosuppression & $18(29.0)^{*}$ & $44(71.0)^{*}$ \\
\hline
\end{tabular}

Note: ${ }^{\star} P<0.05$ compared to the control group

decrease in the test group was much larger than that in the control group $(p<0.05$; Table 2).

\section{Changes in KPS score}

A total of 8 cases in the test group and 3 cases in the controls had improved scores, and 25 cases in the test group and 20 cases in the controls had stable scores. The improvement rate (number of cases with improved scores/total number of cases + number of cases with stable scores/total number of cases) of the test group and the control groups was $53.20 \%$ and $37.10 \%$, respectively $(p<0.05$; Figure 1$)$.

\section{Incidence of adverse reactions}

No patient stopped treatment because of adverse reactions during treatment. After treatment, the vital signs of all patients were stable, and biochemical indices of hepatic and renal function showed no obvious changes. Most of the chemotherapy-associated toxic and side effects such as haematological toxicity, abnormal liver function, urinary system toxicity, and allergy were grade 0 . The reduction in white blood cells and myelosuppression significantly differed in the two groups $(p<0.05$; Table 2$)$.

\section{DISCUSSION}

Malignant ascites is the abnormal accumulation of ascites induced by a malignant tumour, which usually indicates the terminal stage of disease. Important aspects of the treatment of terminal tumours are how to reduce the uncomfortable symptoms induced by ascites and improve the patient's quality of life $[11,12]$.

Current treatments for malignant ascites include symptomatic and supportive treatment, diuresis, puncture and drainage, intra-abdominal drug perfusion, immunotherapy, and anti- 
angiogenesis treatment. However, these treatments all have limitations and the overall outcomes are poor. Recently, thermal therapy has been used extensively [13]. Thermal therapy kills tumour cells by heating tissues to a temperature that will kill tumours cells without damaging normal cells; it is called a green therapy because it is a physical method $[14,15]$.

CHPP has emerged with advances in thermal therapy and the relevant instruments. CHPP combines the anatomical characteristics of the abdominal cavity, the pharmacokinetic characteristics of regional chemotherapeutics, and the principles of thermal therapy $[16,17]$. High temperatures can kill tumour cells directly and promote the diffusion of drugs. The synergistic effects of thermal therapy and chemotherapy can kill free tumour cells and microscopic lesions in the abdominal cavity and control ascites. It is much better at controlling peritoneal metastasis than other methods. The application of CHPP renders uncontrollable, recurrent, or locally advanced tumours more controllable, without increasing acute or late complications [18].

In this study, the response rate to CHPP for the treatment of tumour-induced malignant ascites was $85.50 \%$, which was higher than that of the systemic intravenous chemotherapy. The improved abdominal distension, reduced ascites, and improved KPS score in the test group were obviously superior to the changes in the control group, which suggests that chemotherapy in combination with CHPP is safe and effective for the treatment of malignant ascites. The optimal temperature for CHPP has not been determined and should be the subject of further studies.

\section{Limitations of the study}

The survival period and long-term curative effects were not investigated because of the short follow-up time. Therefore, the number of cases should be increased and the follow-up time should be extended in future studies to clarify the results. In addition, the effects of drug concentration and perfusion temperature on patients with different constitutions and stages may significantly differ. These should be individualised to the patient's condition to reduce the incidence of abdominal distension, pain, or peritonitis. Moreover, the selection of anti-tumour chemotherapeutics should be specific, because malignant ascites can be induced by multiple tumours and tumours in different sites show different sensitivities to different chemotherapeutics.

\section{CONCLUSION}

CHPP can remarkably improve the short-term curative effects on malignant ascites and enhance the quality of life of patients, without increasing toxic and side effects. Thus, it seems to be a safe and effective therapeutic approach for treating malignant ascites, but further studies are required ascertain this.

\section{DECLARATIONS}

\section{Acknowledgement}

The authors sincerely thank all who supported this work.

\section{Conflict of Interest}

No conflict of interest associated with this work.

\section{Contribution of Authors}

The authors declare that this work was done by the authors named in this article and all liabilities pertaining to claims relating to the content of this article will be borne by them.

\section{Open Access}

This is an Open Access article that uses a funding model which does not charge readers or their institutions for access and distributed under the terms of the Creative Commons Attribution License (http://creativecommons.org/licenses/by/ 4.0) and the Budapest Open Access Initiative (http://www.budapestopenaccessinitiative.org/rea d), which permit unrestricted use, distribution, and reproduction in any medium, provided the original work is properly credited.

\section{REFERENCES}

1. Aslam N, Marino CR. Malignant ascites: new concepts in pathophysiology, diagnosis, and management. Arch Intern Med 2001; 161(22): 2733-2737.

2. Sun YF. Clinical effect of intraperitoneal hyperthermic perfusion combined with systemic chemotherapy in ovarian cancer malignant ascites. Chin $J$ Clin Oncol Rehabil 2013; (4): 369-370.

3. Valle M, Van der Speeten K, Garofalo A. Laparoscopic hyperthermic intraperitoneal peroperative chemotherapy (HIPEC) in the management of refractory malignant ascites: A multi-institutional retrospective analysis in 52 patients. J Surg Oncol 2009; 100(4): 331-334.

4. Shimizu T, Sonoda H, Murata S, Takebayashi K, Ohta $H$, Miyake T, Mekata E, Shiomi H, Naka S, Tani T. Hyperthermic intraperitoneal chemotherapy using a 
combination of mitomycin C,5-fluorouracil, and oxaliplatin in patients at high risk of colorectal peritoneal metastasis: A Phase I clinical study. Eur J Surg Oncol 2014; 40(5): 521-528.

5. Zhang ZS, Xue CN, Zhang J, Zhang $H Q$, Ba N, Xia XZ, Wu $M$, Fan $Q X$. Intraperitoneal hyperthermal chemotherapy with cisplatin combined with capecitabine in the treatment for ascites in patients with advanced gastric carcinoma. J Pract Med 2012; 28(20): 34473449.

6. Fleming ND, Alvarez-Secord A, Von Gruenigen $V$, Miller MJ, Abernethy AP. In-dwelling catheters for the management of refractory malignant ascites: a systematic literature overview and retrospective chart review. J Pain Symptom Manag 2009; 38(3): 341-349.

7. Wu TY, Zhang JQ, Liu YL, Xiao ZZ, Wu ZG, Feng YG, Chen J, Han $X L$. Clinical observation on intraperitoneal perfusion for malignant peritoneal effusion. Cancer Res Clin 2013; 25(3):202-203.

8. Declaration of Helsinki. The 59th World Medical Conference, 2008.

9. Haney JC, Pappas TN. Necrotizing Pancreatitis: diagnosis and management. Surg Clin North Am 2007; 87(6): 1431-1446.

10. National Cancer Institute. Cancer Therapy Evaluation Program: Common Toxicity Criteria. Version 2.0 and 3.0, 1999 and 2006.

11. Smith EM, Jayson GC. The current and future management of malignant ascites. Clin Oncol ( $R$ Coll Radiol) 2003; 15(2): 59-72.
12. Woopen H, Sehouli J. Current and future options in the treatment of malignant ascites in ovarian cancer. Anticancer Res 2009; 29(8): 3353-3359.

13. Raut $C P$, Evans $D B$, Crane $C H$, Pisters PW, Wolff RA. Neoadjuvant therapy for resectable pancreatic cancer. Surg Oncol Clin N Am 2004; 13(4): 639-661.

14. Ba MC, Cui SZ, Lin $S Q$, Tang $Y Q$, Wu YB, Wang $B$, Zhang XL. Chemotherapy with laparoscope-assisted continuous circulatory hyperthermic intraperitoneal perfusion for malignant ascites. World J Gastroenterol 2010; 16(15): 1901-1907.

15. Shido A, Ohmura S, Yamamoto K, Kobayashi T, Fujimura $T$, Yonemura $Y$. Does hyperthermia induce peritoneal damage in continuous hyperthermic peritoneal perfusion World J Surg 2000; 24(5): 507-511.

16. Di Giorgio A, Naticchioni E, Biacchi D, Sibio S, Accarpio F, Rocco M, Tarquini S, Di Seri M, Ciardi A, Montruccoli $D$, Sammartino P. Cytoreductive surgery (peritonectomy procedures) combined with hyperthermic intraperitoneal chemotherapy (HIPEC) in the treatment of diffuse peritoneal carcinomatosis from ovarian cancer. Cancer 2008; 113(2): 315-325.

17. Wang $Y$, Cui SZ, Ba MC. The clinical application of $B$ ultrasound positioning puncture and cathetering based hyperthermic intraperitoneal chemotherapy in the treatment of malignant ascites. J Pract Med 2014; 30 (8): 1273-1275.

18. Li Z, Mi DH, Yang KH, Cao N, Wen ZZ, Tian JH, Jiang L, Bai ZG. Hyperthermic intraperitoneal chemoperfusion for malignant ascites: a systematic review. Chin J Gener Surg 2014; 23(8): 1106-1111. 